\title{
Reliability Analysis with Multiple Dependent Features from a Vibration-Based Accelerated Degradation Test
}

\author{
Fuqiang Sun, ${ }^{1,2}$ Jingcheng Liu, ${ }^{1}$ Xiaoyang Li, ${ }^{1}$ and Haitao Liao ${ }^{2}$ \\ ${ }^{1}$ Science and Technology on Reliability and Environmental Engineering Laboratory, School of Reliability and Systems Engineering, \\ Beihang University, Beijing 100191, China \\ ${ }^{2}$ Department of Industrial Engineering, University of Arkansas, Fayetteville, AR 72701, USA
}

Correspondence should be addressed to Haitao Liao; liao@uark.edu

Received 14 July 2016; Accepted 27 October 2016

Academic Editor: Minvydas Ragulskis

Copyright (C) 2016 Fuqiang Sun et al. This is an open access article distributed under the Creative Commons Attribution License, which permits unrestricted use, distribution, and reproduction in any medium, provided the original work is properly cited.

\begin{abstract}
Accelerated degradation testing (ADT) has been widely used for reliability prediction of highly reliable products. In many applications, ADT data consists of multiple degradation-related features, and these features are usually dependent. When dealing with such ADT data, it is important to fully utilize the multiple degradation features and take into account their inherent dependency. This paper proposes a novel reliability-assessment method that combines Brownian motion and copulas to model ADT data obtained from vibration signals. In particular, degradation feature extraction is first carried out using the raw vibration signals, and a feature selection method quantifying feature properties, such as trendability, monotonicity, and robustness, is adopted to determine the most suitable degradation features. Then, a multivariate s-dependent ADT model is developed, where a Brownian motion is used to depict the degradation path of each degradation feature and a copula function is employed to describe the dependence among these degradation features. Finally, the proposed ADT model is demonstrated using the vibration-based ADT data for an electric motor.
\end{abstract}

\section{Introduction}

Nowadays, products are made more reliable due to the advances of design and manufacturing and to the improvement of materials technology. As a result, traditional reliability analysis methods based on lifetime data are becoming less capable of evaluating the reliability of such highly reliable products. In practice, the failure mechanism of a product often can be traced to the degradation of the product's key performance parameters, such as wear, fatigue crack, and material corrosion. The product is considered to be failed if any of the performance parameters exceeds its critical value. Therefore, an alternative way for evaluating the product's reliability is to analyze the degradation data obtained from a degradation test. To collect degradation data in a short time, accelerated degradation testing (ADT) technique has been proposed, where some units of the product are exposed to harsher-than-normal conditions in order to speed up product degradation. The resulting ADT data will be used to develop an ADT model that enables the extrapolation of the product's reliability under the normal operating conditions.

In order to find the degradation trend of a product and predict its lifetime using $\mathrm{ADT}$, the product's performance indicators must be defined first. Such indicators may include not only the product's functional or performance parameters, but also those special features extracted from raw sensor data [1], such as vibration, force and acoustic signals, temperature, and voltage. In particular, vibration monitoring has been widely used in fault diagnostics and remaining useful life (RUL) prediction for mechanical and electromechanical systems [2]. Nectoux et al. [3] developed a bearing experimental platform, called PRONOSTIA, to perform bearing ADT, where both vibration and temperature signals were monitored. Liu et al. [4] introduced the confidence value $(\mathrm{CV})$ as the degradation index for a product and used a fuzzy regression model to analyze the bearing vibration data given in [3]. However, in the field of prognostic and health management (PHM), little effort has been focused on 
the mathematical modeling of multiple degradation features obtained from vibration-based ADT.

Generally, raw vibration data may not be directly used for degradation modeling. As a solution, signal processing techniques are usually utilized to extract some features from the vibration data [5]. It is not uncommon that quite a few features can be extracted in the time, frequency, and timefrequency domains as well as via information entropy analysis [3]. However, since not all features are required in describing the product's degradation process, it is necessary to perform dimension reduction for the purpose of degradation modeling. There are two popular ways to do that. The first one is to perform principal component analysis, singular value decomposition, self-organizing map, or clustering [2]. The other one is feature selection that selects a subset of features by assessing the fitness of candidate features [6-10]. The purpose of performing feature selection is to identify those features that can best describe the fault progression process [8]. Coble and Hines [6] proposed three metrics for feature selection, that is, trendability, monotonicity, and prognosability. Javed et al. [9] performed feature selection by evaluating feature fitness using the monotonicity metric defined in [6] and a new trendability metric, and Liao [8] only used the monotonicity criterion. Camci et al. [10] divided a degradation feature into segments and the average separability of all segments was defined for feature selection. Zhang et al. [7] defined three other metrics, that is, correlation, monotonicity, and robustness, for selecting more relevant degradation features.

It is quite common that there is an s-dependent relationship among the stochastic processes describing different degradation features. Clearly, ignoring such dependency in ADT analysis will lead to inaccurate reliability estimates. For modeling multiple degradation processes, most work considers an independence assumption or multivariate normal distributions [11, 12]. However, these assumptions may not be appropriate in many engineering applications. Copulas provide a convenient way to model the dependence of random variables characterized by their marginal distributions [13]. In addition, no constraints on the univariate marginal distributions are required in implementing copula. Because of its advantages, copula has become a popular approach in multivariate statistical analysis. Sari et al. [14] used a copula function to describe the correlation between two performance characteristics of light emitting diode (LED) and combined it with a generalized linear regression model to model bivariate constant-stress ADT. Pan et al. [15] discussed the bivariate degradation modeling approaches based on Wiener processes and copulas under constantstress accelerating scenarios as well. Similarly, Peng et al. [16], Liu et al. [17], Wang and Pham [18], and Li and Xue [19] also employed the copula method to model the dependency between bivariate degradation features. However, previous research is limited on modeling the dependence between bivariate degradation processes, and little effort has been focused on multiple dependent degradation processes.

Another key issue in ADT modeling is how to choose appropriate mathematic models to depict the degradation paths. In the literature, two kinds of degradation models that have been widely used are stochastic process model (e.g., Brownian motion, gamma process, and inverse Gaussian process) and general path model [20]. Ye and Xie [20] conducted a comprehensive literature review on Brownian motion based degradation models. Liao and Elsayed [21] developed an ADT model based on Brownian motion to predict the field reliability of LED considering stress variations. Guan et al. [22] adopted Brownian motion to model the constant-stress ADT (CSADT) and estimated the model parameters using an objective Bayesian method.

Based on the related literature, one can see that some technical problems have not been resolved in the study of vibration-based ADT with multiple features, including fitness analysis of degradation indicators, development of multivariate s-dependent ADT model, and parameter estimation. To overcome these challenges, a novel reliability modeling method for vibration-based CSADT with multiple dependent features is proposed in this paper. To the best of our knowledge, this study is the first attempt in the related areas. The remainder of this paper is organized as follows. The framework of the proposed method is given in Section 2. Section 3 introduces the feature exaction and selection methods. Section 4 provides a multivariate ADT model based on copulas. In Section 5, a case study is used to illustrate the validity of the proposed model. Finally, conclusions are drawn in Section 6.

\section{Framework of the Proposed Model}

Figure 1 provides the proposed framework for reliability and life evaluation using vibration-based ADT data. The framework consists of two parts: fitness analysis of degradation indicators and modeling of multivariate ADT data.

The first part is to extract and select the degradation indicators from the raw vibration data. It is often necessary to first denoise the vibration data to reduce the negative influence of environmental noise on the subsequent analysis. Then, features are extracted in four domains, that is, time, frequency, time-frequency, and information domains. Since the dimension of the features is high, three goodness metrics are used for the selection of the most suitable degradation indicators from these candidate features.

The other part is to model the multiple accelerated degradation processes describing the selected features. At first, a Brownian motion model is adopted to depict the degradation path of each feature and to extrapolate the reliability related to each feature under the normal stress with an acceleration model and a specified failure threshold. Afterwards, a copula function is used to describe the dependence of different degradation features resulting in a multivariate s-dependent ADT model. A two-stage statistical inference procedure, called Inference Functions for Margins (IFM) method, is employed to estimate the unknown parameters of the proposed model. Finally, Akaike information criterion (AIC) is used to determine the best fitting copula function, and the system joint reliability under the normal operating condition can be obtained.

The following sections will elaborate on the analysis of vibration-based ADT with multiple features. 


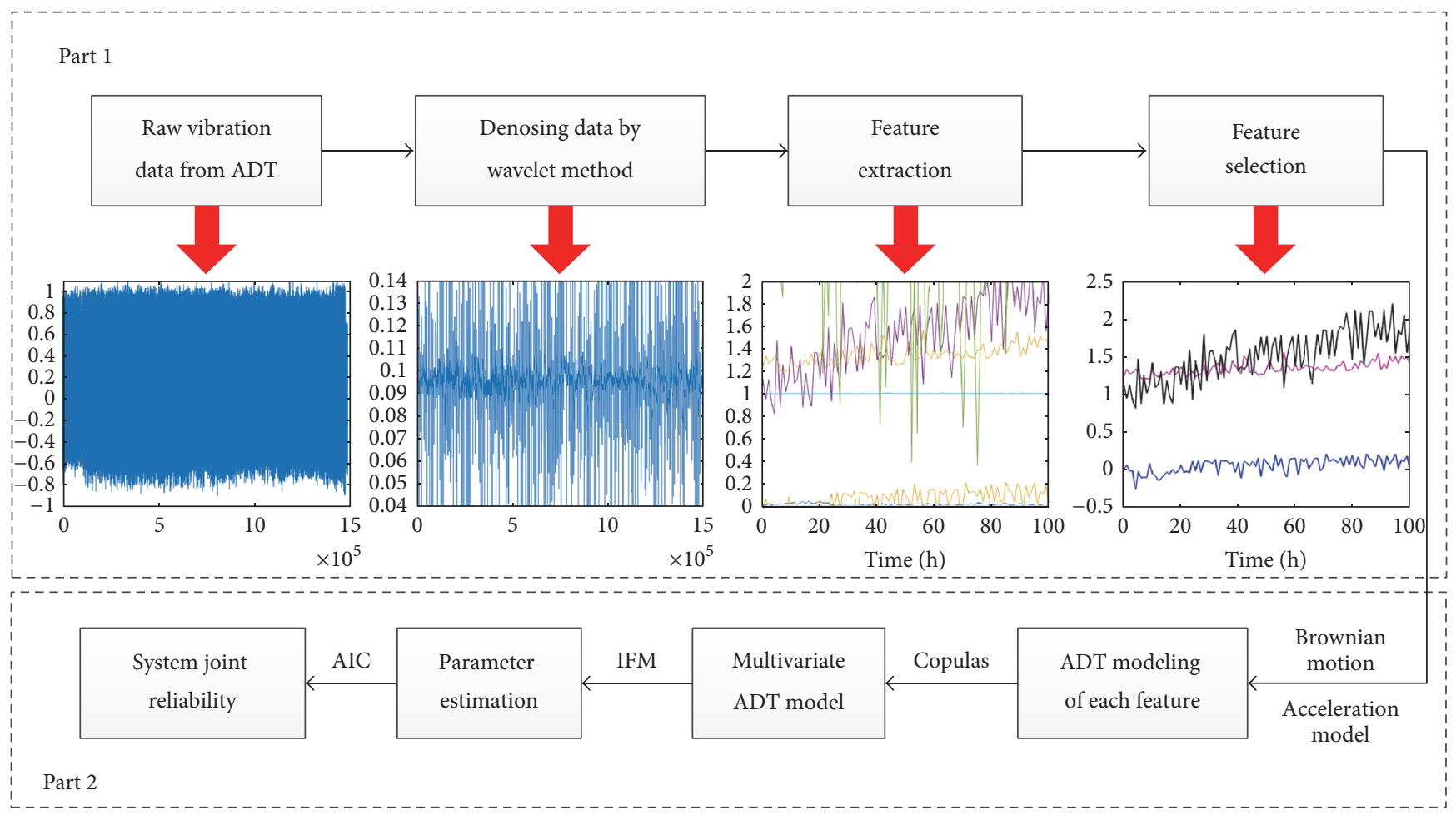

FIGURE 1: The framework of reliability and life evaluation for vibration-based ADT.

\section{The Degradation Indicator Fitness Analysis}

3.1. Signal Processing and Feature Extraction. Denoising is an important step in effective fault detection, diagnosis, and prognostics [23]. In this paper, wavelet automatic denoising technique [24] is used to remove the noise from vibration signals prior to feature extraction. This technique is capable of deconstructing complex signals into basis signals of finite bandwidth and then reconstructing them again with very little loss of information, which is best for nonstationary signal analysis.

Then, different signal processing methods are utilized to find the most valuable features relevant to the product's performance state. Indeed, feature extraction via signal processing is considered one of the major elements and the most challenging aspect in analyzing vibration-based ADT data [25]. The existing signal processing and feature extraction techniques dealing with vibration signals mainly include four categories [2].

(1) Time Domain. Time domain feature extraction is performed directly on the waveforms of vibration signals, which are suitable for fault detection. Usually, the extracted features include mean, variance, waveform factor, crest factor, clearance factor, and kurtosis factor.

(2) Frequency Domain. Frequency domain analysis can identify and isolate those important frequency components of the signals. The most popular method is the fast Fourier transform, with which features such as center frequency, mean square frequency, and frequency variance can be obtained.

(3) Time-Frequency Domain. Time-frequency analysis aims at investigating the signals in both time and frequency domains [9]. In particular, Hilbert-Huang Transform (HHT), based on empirical mode decomposition, is the most powerful technique for the analysis of nonstationary signals [26]. HHT marginal energy is calculated as the feature in this domain.

(4) Information Entropy. Information entropy is a measure of uncertainty of signals [4]. A higher value indicates a higher uncertainty of the signal. Following this idea, the Hilbert marginal energy spectrum entropy is computed in feature extraction.

Let $x_{1}, x_{2}, \ldots, x_{N}$ be the recorded vibration signals, $f$ be the frequency components of the signals, and $S(f)$ be the power spectrum. Table 1 gives various features obtained by processing the original vibration signals.

3.2. Feature Selection. The selection of a subset of appropriate features is essential for prognostic modeling. It is often performed on the basis of feature characteristics by comparing candidate features with respect to their fitness. In other words, the features with high attribute values, such as monotonicity, correlation, trendability, and robustness, should lead to more accurate prognostic results and thus be included for further degradation modeling.

Technically, after removing unwanted noise, a feature is decomposed into its trend and random part by applying a 
TABLE 1: Candidate degradation features.

\begin{tabular}{|c|c|c|c|}
\hline Feature & Formula & Feature & Formula \\
\hline Mean & $\bar{x}=\frac{1}{N} \sum_{i=1}^{N} x_{i}$ & Clearance factor & $L=\frac{\max \left\{\left|x_{i}\right|\right\}}{\left[(1 / N) \sum_{i=1}^{N} \sqrt{\left|x_{i}\right|}\right]^{2}}$ \\
\hline Variance & $\sigma^{2}=\frac{1}{N} \sum_{i=1}^{N}\left(x_{i}-\bar{x}\right)^{2}$ & Frequency center & $\mathrm{FC}=\frac{\int_{0}^{+\infty} f S(f) d f}{\int_{0}^{+\infty} S(f) d f}$ \\
\hline Crest factor & $C=\frac{\max \left\{\left|x_{i}\right|\right\}}{\sqrt{(1 / N) \sum_{i=1}^{N} x_{i}^{2}}}$ & Frequency variance & $\mathrm{VF}=\frac{\int_{0}^{+\infty}(f-\mathrm{FC})^{2} S(f) d f}{\int_{0}^{+\infty} S(f) d f}$ \\
\hline Kurtosis factor & $\alpha=\frac{1}{N} \sum_{i=1}^{N} \frac{\left(x_{i}-\bar{x}\right)^{4}}{\sigma^{4}}$ & HHT marginal energy (HME) & $\begin{array}{c}\operatorname{HME}(\omega)=\int_{0}^{T} H^{2}(\omega, t) d t \\
H(\omega, t)=\operatorname{Re} \sum_{j=1}^{m} c_{j}(t) e^{i \int 2 \pi \omega_{j}(t) d t}\end{array}$ \\
\hline Waveform factor & $S=\frac{\sqrt{(1 / N) \sum_{i=1}^{N} x_{i}^{2}}}{(1 / N) \sum_{i=1}^{N}\left|x_{i}\right|}$ & $\begin{array}{l}\text { HHT marginal energy spectrum } \\
\text { entropy (HMESE) }\end{array}$ & $\begin{aligned} & \mathrm{HMEE}=\frac{-\sum_{j=1}^{m} p_{j} \log _{2} p_{j}}{\log _{2} m} \\
& p_{j}= \frac{\operatorname{HME}(j)}{\sum_{j=1}^{m} \operatorname{HME}(j)} \\
& \sum_{j=1}^{m} p_{j}=1\end{aligned}$ \\
\hline
\end{tabular}

Note: $m$ is the number of frequency components in Hilbert-Huang Transform (HHT).

robust locally weighted scatter plot smooth (RLOESS) with a span value of 0.5 (i.e., $50 \%$ ), as shown in (1). Unlike locally weighted scatter plot smooth (LOESS) that is a popular smoothing method based on a locally weighted regression function and a quadratic polynomial, RLOESS uses a robust regression weight function to make the LOESS process insensitive to outliers

$$
x=x_{T}+x_{R}
$$

where $x$ is the actual feature value, $x_{T}$ is its trend value, and $x_{R}$ is the residual.

Then, a set of mathematical criteria in favor of the features with high attribute values are considered in feature selection.

(1) Monotonicity characterizes the underlying increasing or decreasing trend of feature. It can be measured by the average absolute difference of derivatives for each feature [6] as follows:

$M=$ mean $\left(\left|\frac{\text { No. of } d / d x>0}{n-1}-\frac{\text { No. of } d / d x<0}{n-1}\right|\right)$,

where $n$ is the number of observations, $d / d x$ is the derivative, and mean represents averaging. The value of $M$ is from 0 to 1 , with $M=1$ indicating highly monotonic features and $M=0$ indicating nonmonotonic features.

(2) Trendability is related to the linear relationship between the feature and the time. Clearly, it shows how the degrading state varies with time. The metric of trendability is given by [9]

$$
T=\frac{\left|n\left(\sum x_{T} \cdot t\right)-\left(\sum x_{T}\right)\left(\sum t\right)\right|}{\sqrt{\left[n \sum x_{T}^{2}-\left(\sum x_{T}\right)^{2}\right]\left[n \sum t^{2}-\left(\sum t\right)^{2}\right]}},
$$

where $T \in[0,1]$ is the absolute correlation coefficient between feature trend $x_{T}$ and time $t$. Obviously, a constant function of feature has zero correlation with time, while a linear function indicates a strong correlation between the feature and the time.

(3) Robustness reflects the tolerance of the feature to outliers [7], which is given by

$$
R=\frac{1}{n} \sum \exp \left(-\left|\frac{x_{R}}{x}\right|\right) \text {. }
$$

One can see that all the three metrics of features are confined in the range of $[0,1]$. Due to the fact that one metric only partially measures the suitability of candidate feature, a weighted sum of the three metrics is used in this paper as the fitness function for feature selection

$$
\begin{array}{ll}
\max & \text { fitness }=w_{1} \cdot M+w_{2} \cdot T+w_{3} \cdot R \\
\text { s.t. } & \omega_{i}>0 \\
& \sum_{i} \omega_{i}=1, \\
& i=1,2,3,
\end{array}
$$

where constants $\omega_{1}, \omega_{2}$, and $\omega_{3}$ control the importance of each metric in the optimization problem.

\section{Modeling of CSADT with Multiple Features}

4.1. The Univariate Accelerated Degradation Model. The premise of ADT-based reliability evaluation is to choose an appropriate model based on the degradation physics or the degradation data [20]. We first focus on each degradation feature. 
4.1.1. Basic Assumptions. Among many stochastic process models, Brownian motion is the most widely used in degradation modeling and analysis. This paper employs this model to describe each degradation process. To evaluate the reliability characteristics of the product under the normal operating condition based on ADT data, the relationship between lifetime characteristics and accelerated stress levels should be modeled. To this end, the following assumptions are made.

(A1) The test specimens are independent, and no catastrophic failures occur during the test.

(A2) Under either the normal condition $S_{0}$ or accelerated conditions $S_{1}<S_{2}<\cdots<S_{K}$, degradation process $Y(t)$ over time can be described by

$$
Y(t)=\sigma B(t)+\mu \cdot t+y_{0},
$$

where $t$ is time, $B(t)$ is a standard Brownian motion, $y_{0}$ is the initial degradation level at time zero, $\mu>0$ is the drift parameter, and $\sigma>0$ is the diffusion parameter describing the variability due to unit-tounit variation and the variation of operating and environmental conditions.

The drift parameter $\mu$, commonly known as the degradation rate, is regarded as a function of stress conditions. Usually, such acceleration relationships, such as Arrhenius model, Eyring model, or inverse power model [27], are obtained from physical mechanism analysis or empirical experiences. In particular, the following log-linear relationship is considered:

$$
\ln \mu=\ln d(S)=a+b \cdot \varphi(S),
$$

where $a$ and $b$ are unknown parameters, $d(S)$ is the degradation rate under stress level $S$, and $\varphi(S)$ is a function of stress level depending on the stress type. For example, if $S$ is absolute temperature, $\varphi(S)=1 / S$; if $S$ is voltage, $\varphi(S)=\ln S$.

(A3) The diffusion parameter $\sigma$ does not change with stress, that is, $\sigma_{0}=\sigma_{1}=\cdots=\sigma_{K}=\sigma$ for the normal condition $S_{0}$ and accelerated conditions $S_{1}<S_{2}<$ $\cdots<S_{K}$.

4.1.2. Reliability Function for Each Degradation Process. Because of the independent increment property of Brownian motion, the degradation increment $\Delta y$ over nonoverlapped interval $\Delta t$ is normally distributed with mean $\mu \Delta t$ and variance $\sigma^{2} \Delta t$. Its probability density function (PDF) is

$$
f(\Delta y)=\frac{1}{\sqrt{2 \pi \sigma^{2} \Delta t}} \exp \left\{-\frac{[\Delta y-\mu \cdot \Delta t]^{2}}{2 \sigma^{2} \Delta t}\right\} .
$$

Generally, a product is supposed to be failed if its performance degradation exceeds a critical threshold for the first time. In other words, the first passage time (FPT) distribution is used as the product's lifetime distribution. For a given critical threshold $D$, the lifetime $T_{D}$ of the product is the instant when degradation process $Y(t)$ exceeds $D$ for the first time; that is,

$$
T_{D}=\inf \{t>0 \mid Y(t) \geq D\} .
$$

It is well-known that the first passage time follows the inverse Gaussian distribution with the following PDF [28]:

$$
f\left(t ; y_{0}, D\right)=\frac{D-y_{0}}{\sigma \sqrt{2 \pi t^{3}}} \exp \left\{-\frac{\left[\left(D-y_{0}\right)-\mu \cdot t\right]^{2}}{2 \sigma^{2} t}\right\} .
$$

Combined with (7), the associated reliability function under normal operational condition $S_{0}$ can be expressed as

$$
\begin{array}{r}
R(t)=\Phi\left[\frac{D-y_{0}-d\left(S_{0}\right) t}{\sigma \sqrt{t}}\right] \\
-\exp \left(\frac{2 d\left(S_{0}\right)\left(D-y_{0}\right)}{\sigma^{2}}\right) \\
\cdot \Phi\left[-\frac{D-y_{0}+d\left(S_{0}\right) t}{\sigma \sqrt{t}}\right],
\end{array}
$$

where $\Phi(\cdot)$ is the cumulative distribution function of standard normal distribution.

\subsection{Use of Copulas for Multiple Features}

4.2.1. Definition and Basic Properties. Sklar's theorem lays the theoretical foundation for copulas and their applications [13].

Theorem 1 (Sklar's theorem [29]). Let $X=\left(X_{1}, \ldots, X_{N}\right)$ be a random vector with marginal distributions $F_{1}\left(x_{1}\right), \ldots, F_{N}\left(x_{N}\right)$ and $F$ be their joint distribution function. Then, there exists a copula function $C$ such that

$$
\begin{aligned}
& F\left(x_{1}, \ldots, x_{n}, \ldots, x_{N}\right) \\
& \quad=C\left(F_{1}\left(x_{1}\right), \ldots, F_{n}\left(x_{n}\right), \ldots, F_{N}\left(x_{N}\right)\right) .
\end{aligned}
$$

If $F_{1}\left(x_{1}\right), \ldots, F_{N}\left(x_{N}\right)$ are continuous, the copula function $C$ is unique. Conversely, if $F_{1}\left(x_{1}\right), \ldots, F_{N}\left(x_{N}\right)$ are univariate distributions, the function $F$ defined by (12) is the joint distribution function associated with margins $F_{1}\left(x_{1}\right), \ldots, F_{N}\left(x_{N}\right)$.

Based on the theorem, a multivariate copula function can be defined as follows.

Definition 2 (n-dimensional copula or $n$-copula [13]). An $N$ dimensional copula is a function $C$ mapping from $\mathbf{I}^{N}=$ $[0,1]^{N}$ to I with the following properties:

(1) Grounded: for every $\mathbf{u}=\left(u_{1}, \ldots, u_{N}\right)$ in $\mathbf{I}^{N}, C(\mathbf{u})=0$ if at least one coordinate of $\mathbf{u}$ is 0 .

(2) Uniform marginals: if all coordinates of $\mathbf{u}$ are ones except $u_{k}$, then

$$
C(\mathbf{u})=C\left(1, \ldots, 1, u_{k}, 1, \ldots, 1\right)=u_{k} .
$$


TABLE 2: Typical multivariate copulas.

\begin{tabular}{lcc}
\hline Copulas & $C\left(u_{1}, u_{2}, \ldots, u_{n}\right)$ & Parameters \\
$\begin{array}{l}\text { Gaussian } \\
\text { copula }\end{array}$ & $\Phi_{\rho}\left[\Phi^{-1}\left(u_{1}\right), \Phi^{-1}\left(u_{2}\right), \ldots, \Phi^{-1}\left(u_{n}\right)\right]$ & $\rho \in(-1,1)$ \\
$\begin{array}{l}\text { Student's } \\
t \text {-copula }\end{array}$ & $t_{\rho, v}\left[t_{v}^{-1}\left(u_{1}\right), t_{v}^{-1}\left(u_{2}\right), \ldots, t_{v}^{-1}\left(u_{n}\right)\right]$ & $\rho \in(-1,1)$ \\
$\begin{array}{l}\text { Clayton } \\
\text { copula }\end{array}$ & $\left(\sum_{i=1}^{n} u_{i}^{-\delta}-n+1\right)^{-1 / \delta}$ & $\delta>0$ \\
$\begin{array}{l}\text { Frank } \\
\text { copula }\end{array}$ & $-\delta^{-1} \log \left\{1+\frac{\prod_{i=1}^{n}\left[\exp \left(-\delta u_{i}\right)-1\right]}{[\exp (-\delta)-1]^{n-1}}\right\}$ & $\delta \in$ \\
$\begin{array}{l}\text { Joe } \\
\text { copula }\end{array}$ & $1-\left\{1-\prod_{i=1}^{n}\left[1-\left(1-u_{i}\right)^{\delta}\right]\right\}^{1 / \delta}$ & $\delta>0$ for $n \geq 3$ \\
\end{tabular}

(3) $N$-increasing: for each hyperrectangle $B=\prod_{i=1}^{N}\left[u_{i}\right.$, $\left.v_{i}\right] \subseteq[0,1]^{N}$, the $C$-volume of $B$ is nonnegative:

$$
\int_{B} d C([\mathbf{u}, \mathbf{v}])=\sum_{\mathbf{z} \in X_{i=1}^{N}\left\{u_{i}, v_{i}\right\}}(-1)^{N(\mathbf{z})} C(\mathbf{z}) \geq 0,
$$

where $N(\mathbf{z})=\#\left\{k: z_{k}=u_{k}\right\}$.

Furthermore, the PDF corresponding to joint distribution function $F\left(x_{1}, \ldots, x_{n}, \ldots, x_{N}\right)$ can be calculated as

$$
\begin{aligned}
f & \left(x_{1}, \ldots, x_{n}, \ldots, x_{N}\right) \\
& =c\left(F_{1}\left(x_{1}\right), \ldots, F_{n}\left(x_{n}\right), \ldots, F_{N}\left(x_{N}\right)\right) \prod_{n=1}^{N} f_{n}\left(x_{n}\right),
\end{aligned}
$$

where $f_{n}(\cdot)$ is the PDF of a marginal distribution $F_{n}(\cdot)$, and $c(\cdot)$ is the density of copula function $C$ :

$$
\begin{aligned}
c\left(u_{1}, \ldots, u_{n}, \ldots, u_{N}\right)= & \frac{\partial^{N} C\left(u_{1}, \ldots, u_{n}, \ldots, u_{N}\right)}{\partial u_{1}, \ldots, \partial u_{n}, \ldots, \partial u_{N}} \\
& \forall \mathbf{u}=\left(u_{1}, \ldots, u_{n}, \ldots, u_{N}\right) \in \mathbf{I}^{N} .
\end{aligned}
$$

4.2.2. Selection of Copula Family. It is important to select an appropriate copula family for the given data, as the selection significantly influences the prediction performance. Table 2 presents five popular multivariate copula families, where $u_{1}, \ldots, u_{n}$ are $n$ random variables.

To balance the statistical fitting and model complexity, the values of classic Akaike information criterion (AIC) are computed for the candidate copula families:

$$
\mathrm{AIC}=2 k-2 \ln L,
$$

where $k$ is the number of estimated parameters in the model, and $L$ is the value of the corresponding maximum likelihood. By comparing the AIC values, the family with the minimum value will be selected.

4.3. Multivariate ADT Model. To analyze the multivariate CSADT data, additional assumptions are considered.
(A4) For a product with multiple degradation indicators, the dependency among the features can be characterized by a copula function.

(A5) The degradation measurements of all specimens are collected at the same time.

(A6) The specimen is considered to be failed if one of the features reaches its corresponding failure threshold for the first time.

Suppose that a product has $P$ degradation features and each of the accelerated degradation processes is modeled by a drift Brownian motion. $Y_{p}(t), p=1,2, \ldots, P$, denotes the degradation process of the $p$ th characteristic index at operating condition $S_{0}$, and its corresponding failure threshold is $D_{p}$. Let the failure time of the $p$ th characteristic index be $T_{p}$, and the lifetime of the product be $T$, so $T=\min \left(T_{1}, \ldots, T_{p}\right)$. Clearly, the system reliability under normal condition $S_{0}$ can be expressed as

$$
\begin{aligned}
R(t) & =P(T>t)=P\left(T_{1}>t, \ldots, T_{P}>t\right) \\
& =P\left(Y_{1}(t)<D_{1}, \ldots, Y_{P}(t)<D_{P}\right)
\end{aligned}
$$

Apparently, if the $P$ degradation features are independent, (18) can be rewritten as

$$
\begin{aligned}
R(t) & =P\left(Y_{1}(t)<D_{1}\right) \times \cdots \times P\left(Y_{P}(t)<D_{P}\right) \\
& =R_{1}(t) \times \cdots \times R_{P}(t),
\end{aligned}
$$

where $R_{p}(t), p=1,2, \ldots, P$ is the $p$ th characteristic index's reliability function under normal operating condition $S_{0}$, as defined in (11). However, this is often not true in many engineering applications. To address the dependence of $P$ degradation features, a copula function can be utilized. In particular, the product's reliability at time $t$ under normal operating condition $S_{0}$ can be performed as

$$
R(t)=C\left(R_{1}(t), R_{2}(t), \ldots, R_{P}(t) ; \boldsymbol{\theta}\right),
$$

where $\boldsymbol{\theta}$ is the parameter set of the copula.

4.4. Parameter Estimation. Consider a copula-based multivariate distribution for the random vector $\left[Y_{1}, \ldots, Y_{P}\right]$, with joint PDF

$$
\begin{aligned}
& f\left(\mathbf{y} ; \boldsymbol{\alpha}_{1}, \ldots, \boldsymbol{\alpha}_{P}, \boldsymbol{\theta}\right) \\
& \quad=c\left(F_{1}\left(y_{1} ; \boldsymbol{\alpha}_{1}\right), \ldots, F_{P}\left(y_{P} ; \boldsymbol{\alpha}_{P}\right) ; \boldsymbol{\theta}\right) \prod_{p=1}^{P} f_{p}\left(y_{p} ; \boldsymbol{\alpha}_{p}\right),
\end{aligned}
$$

where $F_{p}\left(\cdot ; \boldsymbol{\alpha}_{p}\right)$ and $f_{p}\left(\cdot ; \boldsymbol{\alpha}_{p}\right), p=1, \ldots, P$, are the $p$ th marginal CDF and PDF with parameter set $\boldsymbol{\alpha}_{p}$, and $c(\cdot ; \boldsymbol{\theta})$ is the density of copula function $C(\cdot ; \boldsymbol{\theta})$ with parameter $\boldsymbol{\theta}$.

For a sample of size $n$, the full log-likelihood function can be expressed as

$$
\ln L\left(\boldsymbol{\alpha}_{1}, \ldots, \boldsymbol{\alpha}_{P}, \boldsymbol{\theta}\right)
$$



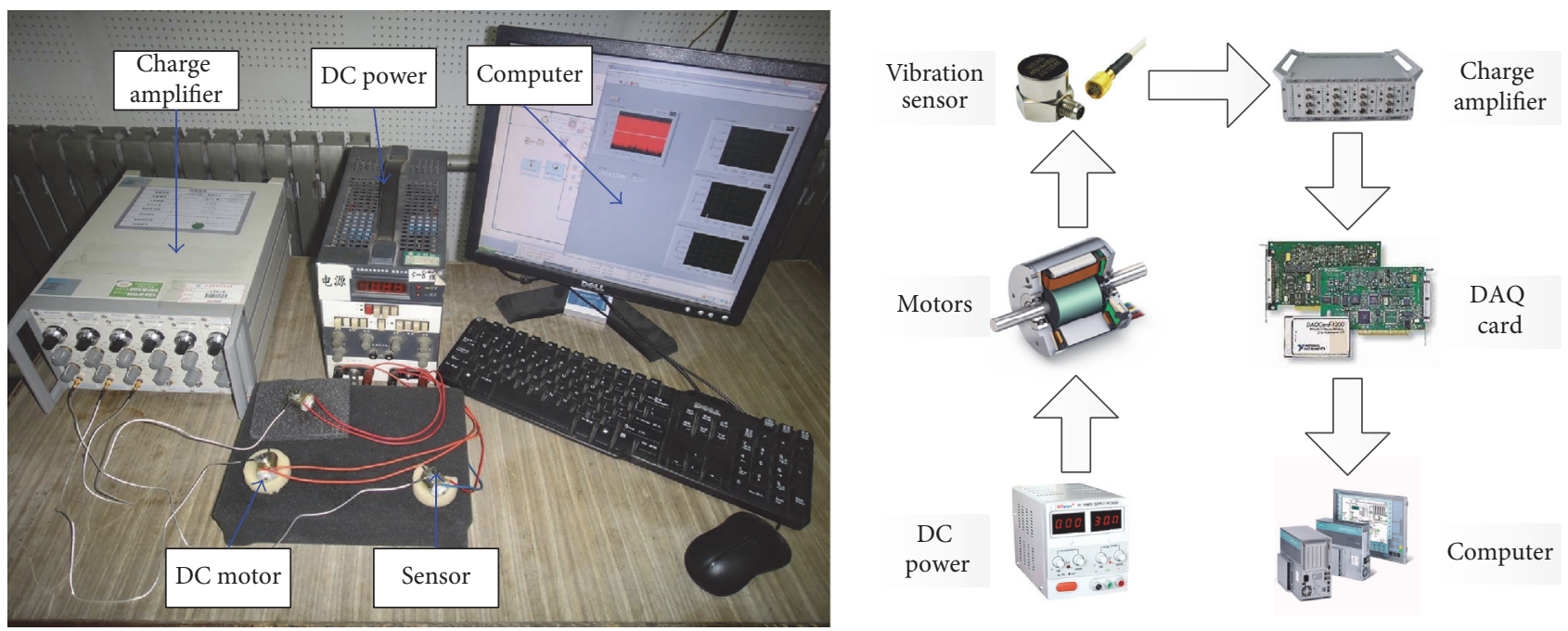

FIgURE 2: Overview of the motor vibration-based ADT platform.

$$
\begin{aligned}
= & \underbrace{\sum_{i=1}^{n} \ln c\left(F_{1}\left(y_{1 i} ; \boldsymbol{\alpha}_{1}\right), \ldots, F_{P}\left(y_{P i} ; \boldsymbol{\alpha}_{P}\right) ; \boldsymbol{\theta}\right)}_{\text {dependence structure } L_{C}} \\
& +\underbrace{\sum_{p=1}^{P} \sum_{i=1}^{n} f_{p}\left(y_{p i} ; \boldsymbol{\alpha}_{p}\right) .}_{\text {marginals } \sum_{p=1}^{P} L_{p}}
\end{aligned}
$$

The maximum likelihood estimates (MLE) of the parameters $\left(\boldsymbol{\alpha}_{1}, \ldots, \boldsymbol{\alpha}_{p}, \boldsymbol{\theta}\right)$ can be obtained by maximizing (22). However, as the dimension of parameters increases, it is difficult to get the optimal solution. Joe [30, 31] proposed the IFM method as a computationally attractive alternative to estimate the parameters in multivariate copula models. Technically, (22) is first decomposed into two parts. The first part, denoted by $L_{C}$, is the contribution from the dependence structure in data, and the other part is the contributions from each margin, $L_{p}, p=1, \ldots, P$. Then, The IFM method separately estimates the parameters of marginal distributions and the parameters of the copula in two stages. In the first stage, the estimates of the marginal distribution parameters are obtained from the $\log$-likelihood $L_{p}$ of each margin. In the second stage, the copula parameter estimates are computed by maximizing $L_{C}$ based on the marginal distribution parameters estimated in the first stage. In this paper, we use this method to estimate the parameters of the proposed multivariate ADT model.
4.4.1. Marginal Distribution Parameters Estimation. Suppose that in a CSADT with $K$ stress levels the total sample size is $n$. There are $n_{l}$ specimens under stress level $S_{l}$. During the CSADT, all specimens are measured once in every $\Delta t$ time interval and there are $M_{l}$ inspections under $S_{l}$. Then, the observation of the $p$ th characteristic index at time $t_{l i j}$ is $y_{p}\left(t_{l i j}\right), p=1, \ldots, P, l=1, \ldots, K, i=1, \ldots, n_{l}, j=1, \ldots, M_{l}$, where $t_{l i j}$ is the time of the $j$ th measurement of the $i$ th unit under the $l$ th stress level. According to (8), the likelihood function of the $p$ th characteristic indices is given by

$$
\begin{array}{r}
L_{p}=\prod_{l=1}^{K} \prod_{i=1}^{n_{l}} \prod_{j=1}^{M_{l}-1} \frac{1}{\sqrt{2 \pi \sigma_{p}^{2} \Delta t}} \\
\cdot \exp \left\{-\frac{\left[\left(y_{p}\left(t_{l i(j+1)}\right)-y_{p}\left(t_{l i j}\right)\right)-\mu_{p l} \cdot \Delta t\right]^{2}}{2 \sigma_{p}^{2} \Delta t}\right\}, \\
p=1, \ldots, P .
\end{array}
$$

Considering the acceleration model given by (7), $\mu_{p l}$ can be expressed as $\mu_{p l}=d_{p}\left(S_{l}\right)=\exp \left(a_{p}+b_{p} \varphi\left(S_{l}\right)\right)$, where $a_{p}$ and $b_{p}$ are unknown parameters of the $p$ th characteristic index. So the model parameter set of the characteristic index is $\boldsymbol{\alpha}_{p}=\left(a_{p}, b_{p}, \sigma_{p}\right), p=1, \ldots, P$. Let $\Delta y_{p l i j}=y_{p}\left(t_{l i(j+1)}\right)-$ $y_{p}\left(t_{l i j}\right)$; then (23) can be expressed as

$$
\ln L=-\frac{1}{2} \sum_{l=1}^{K} \sum_{i=1}^{n_{l}} \sum_{j=1}^{M_{l}-1}\left\{\ln \left(2 \pi \sigma_{p}^{2} \Delta t\right)+\frac{\left[\Delta y_{p l i j}-\exp \left(a_{p}+b_{p} \varphi\left(S_{l}\right)\right) \cdot \Delta t\right]^{2}}{\sigma_{p}^{2} \Delta t}\right\}, \quad p=1, \ldots, P
$$

and the parameter set $\boldsymbol{\alpha}_{p}=\left(a_{p}, b_{p}, \sigma_{p}\right)$ of each marginal distribution can be estimated by maximizing the loglikelihood function.
4.4.2. Copula Parameter Estimation. By replacing the marginal parameters $\boldsymbol{\alpha}_{p}, p=1, \ldots, P$, with their first-stage estimate $\widehat{\boldsymbol{\alpha}}_{p}$, the copula parameters can be estimated by 

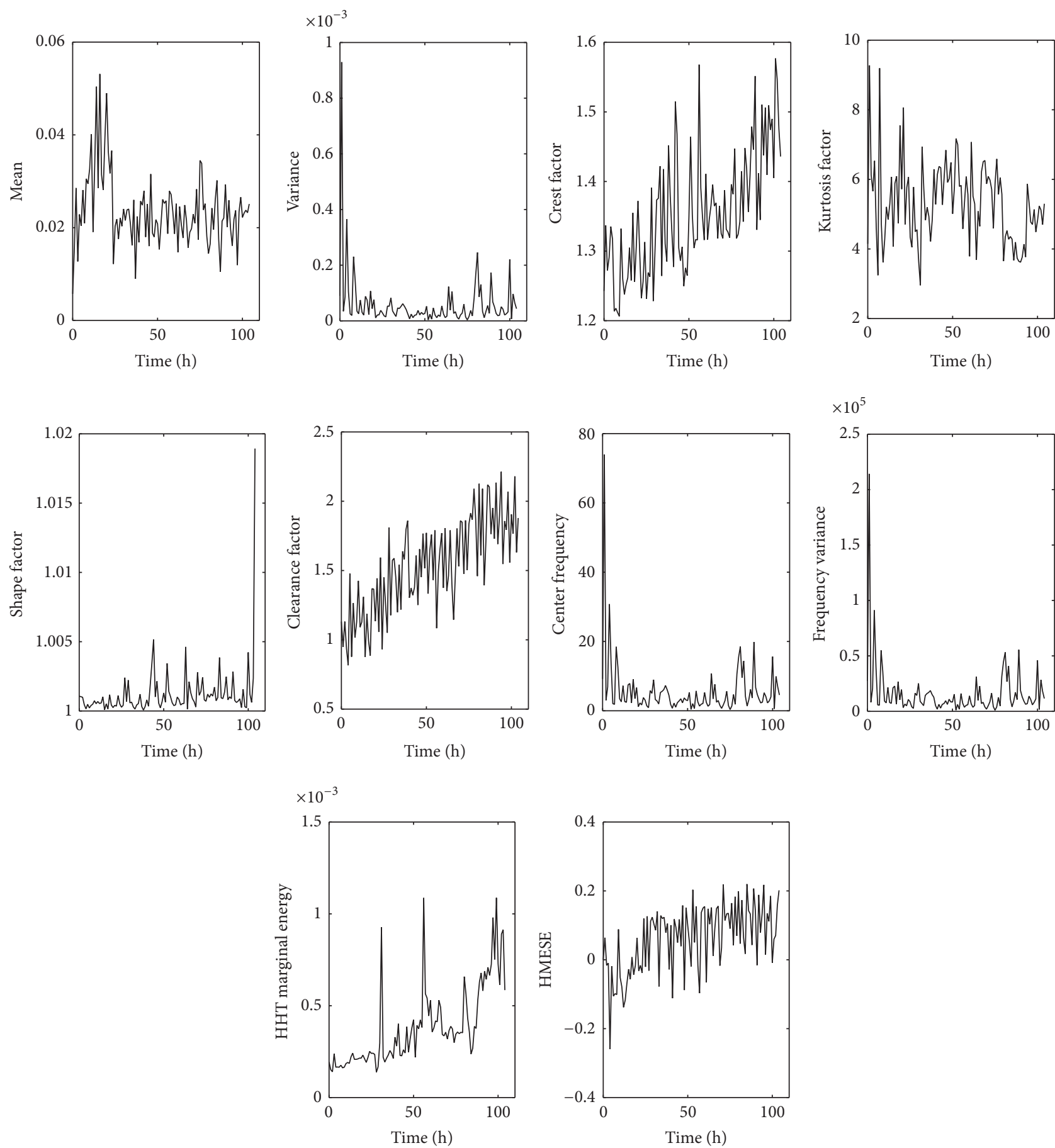

FIgURE 3: Feature set of a motor.

maximizing the copula likelihood contribution. Then, the copula parameter $\boldsymbol{\theta}$ in (20) is given by

$$
=\arg \max \sum_{p=1}^{P} \sum_{i=1}^{n} \ln c\left(R_{1}\left(y_{1 i} ; \widehat{\boldsymbol{\alpha}}_{1}\right), \ldots, R_{P}\left(y_{p i} ; \widehat{\boldsymbol{\alpha}}_{P}\right) ; \boldsymbol{\theta}\right) .
$$$$
\widehat{\boldsymbol{\theta}}
$$

In addition, the AIC method mentioned in Section 4.2.2 is used to quantitatively select the best fitting model from candidate copula functions.

\section{Case Study}

A case study is provided in this section to demonstrate the use of the proposed framework in real-world industrial applications.

5.1. Description of the ADT Experiment. DC motor converts DC power into mechanical energy. It is a key part of robots, electric vehicle, numerical control machines and household appliances, and so forth. The failure of motors 


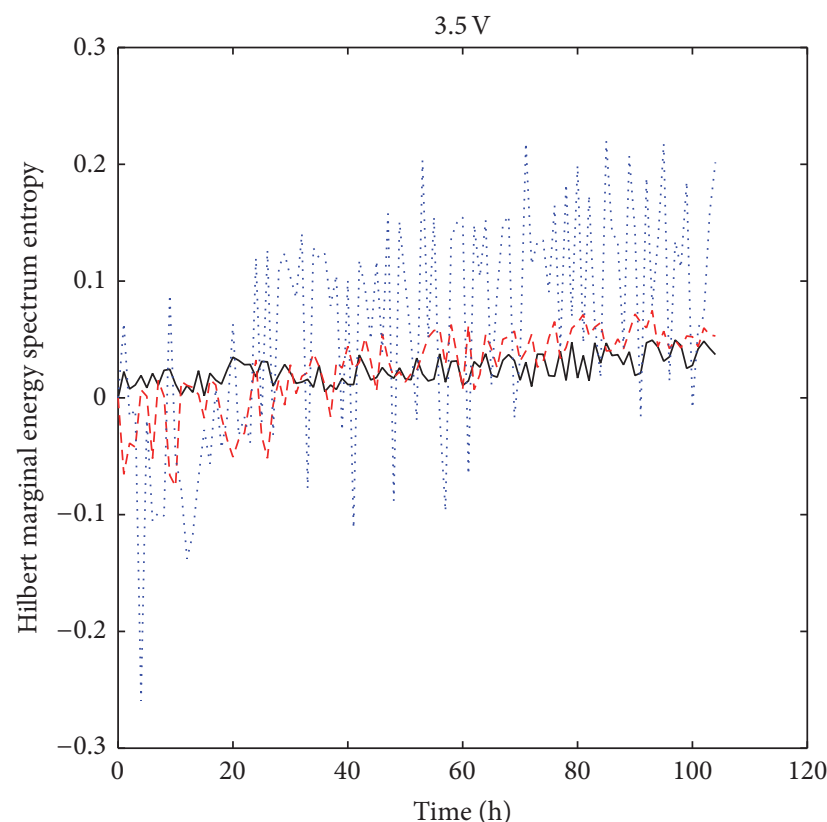

Motor number 11

- - - Motor number 12

Motor number 13

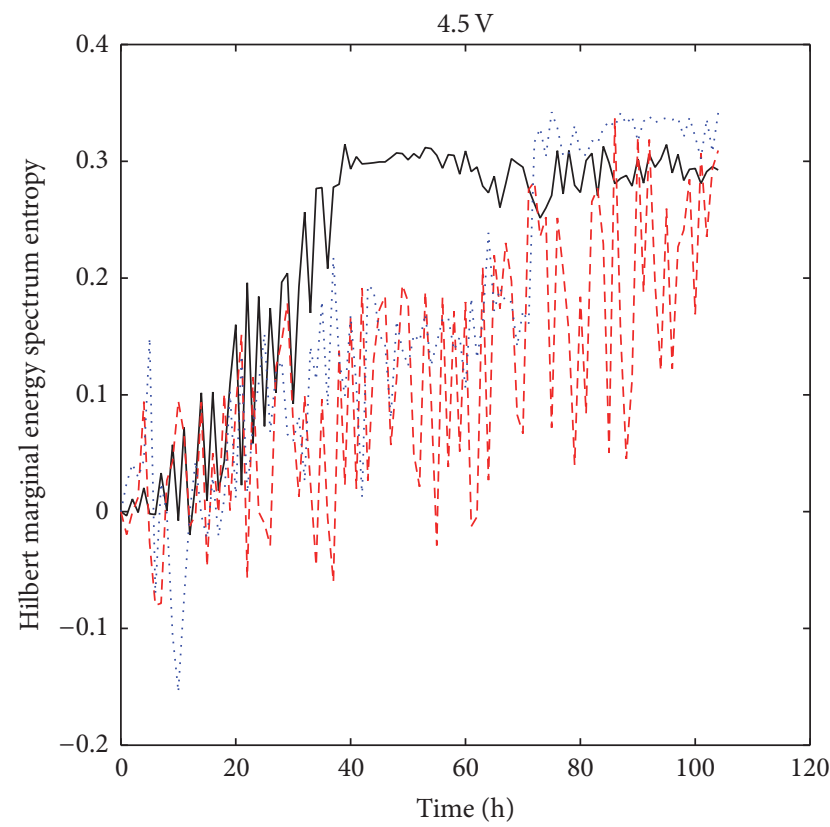

- Motor number 21

- - - Motor number 22

Motor number 23

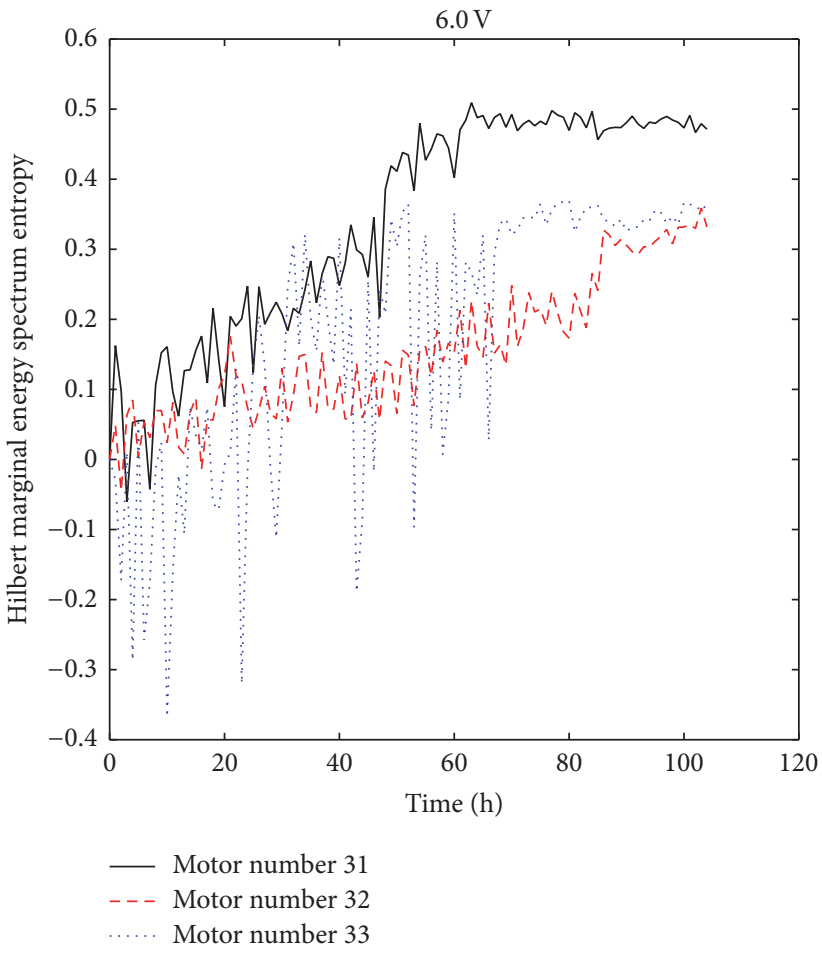

FIGURE 4: The CSADT data of Hilbert marginal energy spectrum entropy.

will significantly decrease the availability and safety of such machines and even lead to big economic losses.

To assess the life and reliability of PZB131 brushed DC motors, a dedicated CSADT platform was designed as shown in Figure 2. The platform is composed of DC power, electric motors, vibration sensors, charge amplifier, data acquisition card, and computer. The key failure mechanism of this type of DC motors is the wear-out between brush and commutator, which is mainly related to the rotating speed and load. To speed up the degradation process, the input voltage was employed as the accelerated stress that controls the rotating speed of motors under a nonload condition. 


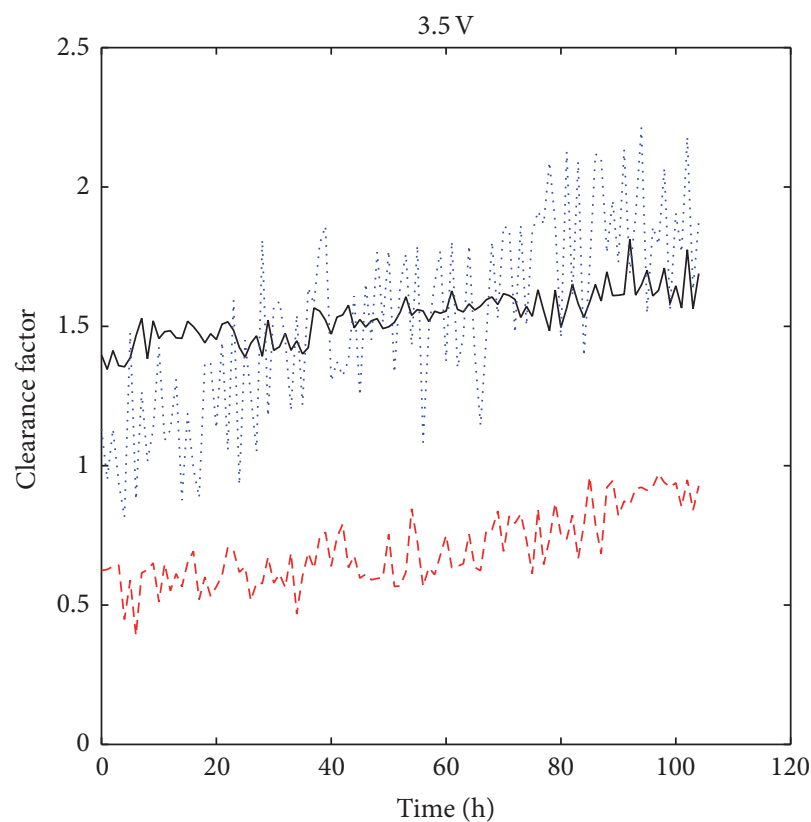

Motor number 11
$\ldots$-... Motor number 12
Motor number 13

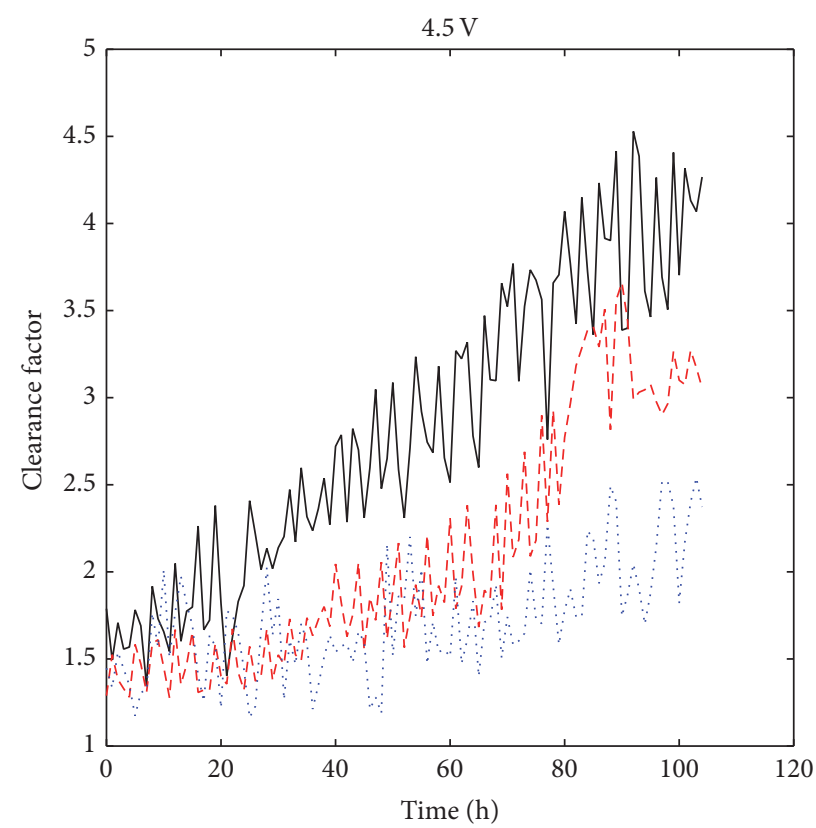

- Motor number 21

- - - Motor number 22

..... Motor number 23

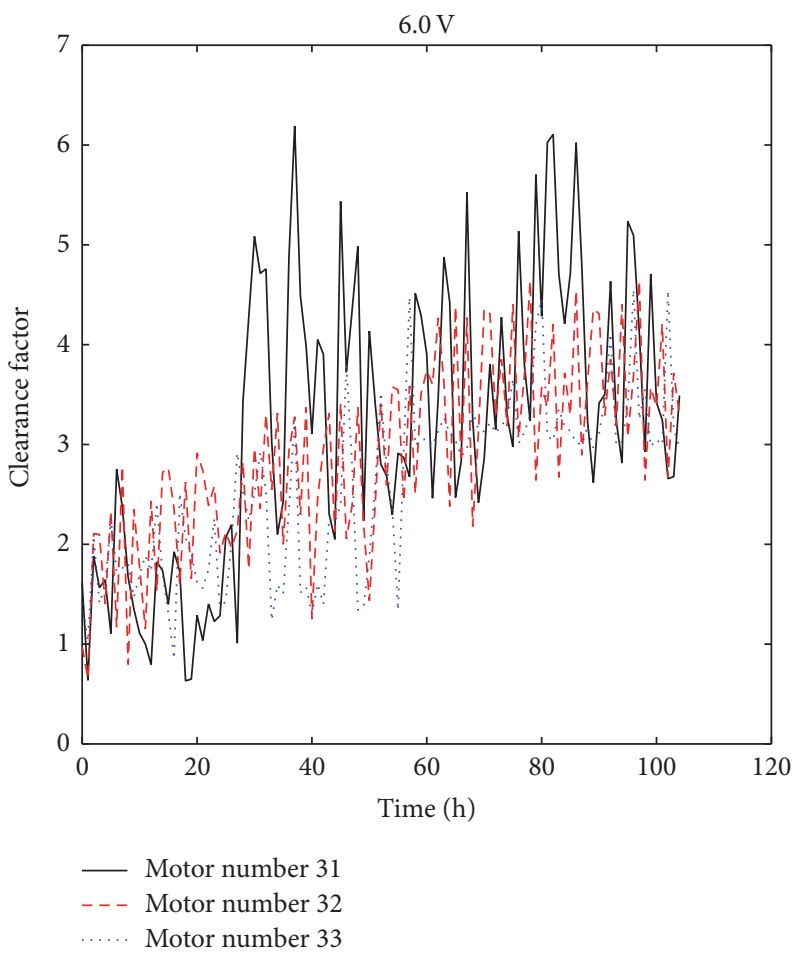

FIGURE 5: The CSADT data of clearance factor.

The rated voltage of the motor is $2.0 \mathrm{~V}$, and the accelerated stress levels are $3.5 \mathrm{~V}, 4.5 \mathrm{~V}$, and $6.0 \mathrm{~V}$. The sample size under each stress level was 3 . The vibration signals on the surface of motor were collected every hour. The sampling time is 2 seconds and the total number of sampling points is 3000 .
5.2. Feature Extraction and Selection. First, denoising of raw vibration signals was performed using the Daubechies (db8) wavelet method based on the principle of Stein's unbiased risk with soft-thresholding [24]. Ten features of each motor were exacted using the signal processing methods addressed in 

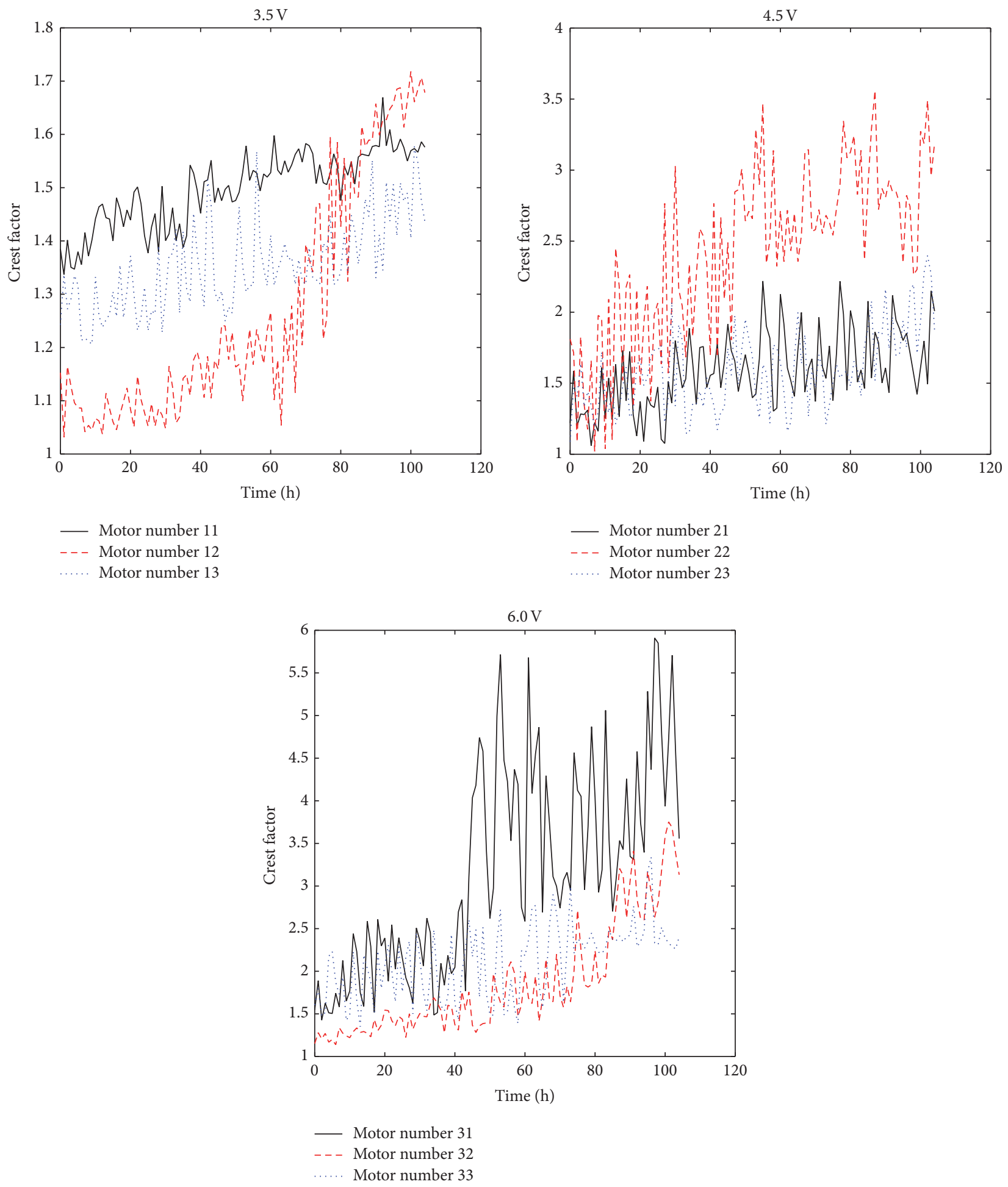

FIGURE 6: The CSADT data of crest factor.

Section 3.1. Figure 3 shows all the candidate features collected from one of the motors.

Afterwards, feature selection was implemented using the proposed feature selecting procedure given in Section 3.2, and the most suitable features were selected from the ten candidate features. Because prognostic features showing significant monotonic trends are more desired [7], different weights, $0.3,0.6$, and 0.1 , were given to trendability, monotonicity, and robustness, respectively. The feature selection results are presented in Table 3 . 

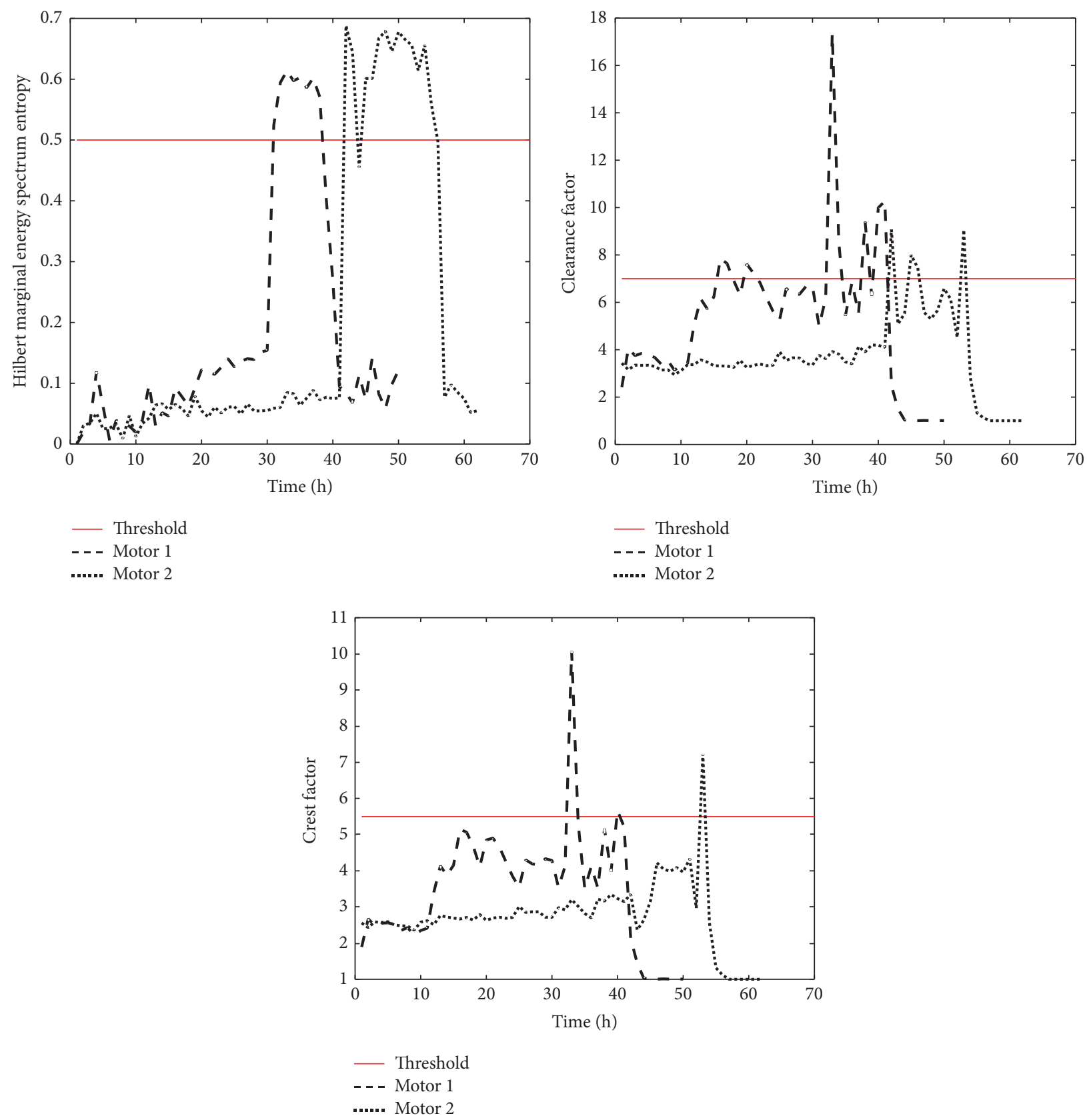

FIgURE 7: Determination of the failure threshold.

Because the clearance factor, crest factor, and HHT marginal energy spectrum entropy (HMESE) fitness were all greater than 0.7 , they were selected as the degradation indicators for the motors. The degradation processes of three selected optimal features are illustrated in Figures 4-6.

5.3. Reliability Modeling. Defining the failure threshold for each feature is the first step for degradation-based reliability modeling. To this end, two motors were tested until failure, and the failure thresholds were determined by analyzing the clearance factor, Hilbert marginal energy spectrum entropy, and crest factor of the motors upon failure. As can be seen in Figure 7 , the relative failure threshold D- $y_{0}$ of Hilbert marginal energy spectrum entropy, clearance factor, and crest factor is $0.5,3$, and 3 , respectively.

Then, the univariate accelerated degradation models for the three features were established, respectively, using the Brownian motion models addressed in Section 4.1. The model parameters were estimated using the method given in Section 4.4.1. Figure 8 shows the reliability functions of the three features calculated by (11). One can see that the reliability curve of crest factor tends to be higher than those of the other two features.

Next, the multivariate ADT model was developed using the copula function that describes the dependence among three features, and the IFM method was used to estimate 
TABLE 3: Feature evaluation results.

\begin{tabular}{|c|c|c|c|c|c|c|c|c|}
\hline No. & Feature & Stress & $T$ & $M$ & $R$ & Fitness & Mean $(f)$ & Rank \\
\hline \multirow{3}{*}{1} & \multirow{3}{*}{ Mean } & $3.5 \mathrm{~V}$ & 0.2491 & 0.5556 & 0.9500 & 0.5425 & \multirow{3}{*}{0.5102} & \multirow{3}{*}{8} \\
\hline & & $4.5 \mathrm{~V}$ & 0.1616 & 0.3333 & 0.9839 & 0.3469 & & \\
\hline & & $6.0 \mathrm{~V}$ & 0.7032 & 0.5556 & 0.9682 & 0.6411 & & \\
\hline \multirow{3}{*}{2} & \multirow{3}{*}{ Variance } & $3.5 \mathrm{~V}$ & 0.6042 & 0.5556 & 0.8549 & 0.6001 & \multirow{3}{*}{0.5350} & \multirow{3}{*}{7} \\
\hline & & $4.5 \mathrm{~V}$ & 0.4839 & 0.3333 & 0.7852 & 0.4237 & & \\
\hline & & $6.0 \mathrm{~V}$ & 0.5634 & 0.5556 & 0.7892 & 0.5813 & & \\
\hline \multirow{3}{*}{3} & \multirow{3}{*}{ Crest factor } & $3.5 \mathrm{~V}$ & 0.8614 & 0.5556 & 0.9808 & 0.6898 & \multirow{3}{*}{0.7509} & \multirow{3}{*}{3} \\
\hline & & $4.5 \mathrm{~V}$ & 0.7359 & 1 & 0.9263 & 0.9134 & & \\
\hline & & $6.0 \mathrm{~V}$ & 0.7430 & 0.5556 & 0.9319 & 0.6494 & & \\
\hline \multirow{3}{*}{4} & \multirow{3}{*}{ Kurtosis factor } & $3.5 \mathrm{~V}$ & 0.3349 & 0.5556 & 0.9438 & 0.5282 & \multirow{3}{*}{0.4236} & \multirow{3}{*}{10} \\
\hline & & $4.5 \mathrm{~V}$ & 0.3380 & 0.3333 & 0.7947 & 0.3809 & & \\
\hline & & $6.0 \mathrm{~V}$ & 0.3179 & 0.3333 & 0.6620 & 0.3616 & & \\
\hline \multirow{3}{*}{5} & \multirow{3}{*}{ Waveform factor } & $3.5 \mathrm{~V}$ & 0.5856 & 0.3333 & 0.9987 & 0.4756 & \multirow{3}{*}{0.6896} & \multirow{3}{*}{4} \\
\hline & & $4.5 \mathrm{~V}$ & 0.7208 & 0.7778 & 0.9962 & 0.7825 & & \\
\hline & & $6.0 \mathrm{~V}$ & 0.8182 & 0.7778 & 0.9858 & 0.8107 & & \\
\hline \multirow{3}{*}{6} & \multirow{3}{*}{ Clearance factor } & $3.5 \mathrm{~V}$ & 0.8603 & 1 & 0.9436 & 0.9525 & \multirow{3}{*}{0.8961} & \multirow{3}{*}{1} \\
\hline & & $4.5 \mathrm{~V}$ & 0.8570 & 1 & 0.9460 & 0.9517 & & \\
\hline & & $6.0 \mathrm{~V}$ & 0.7649 & 0.7778 & 0.8804 & 0.7842 & & \\
\hline \multirow{3}{*}{7} & \multirow{3}{*}{ Frequency center } & $3.5 \mathrm{~V}$ & 0.6258 & 0.5556 & 0.8631 & 0.6074 & \multirow{3}{*}{0.6260} & \multirow{3}{*}{5} \\
\hline & & $4.5 \mathrm{~V}$ & 0.6474 & 0.5556 & 0.7387 & 0.6014 & & \\
\hline & & $6.0 \mathrm{~V}$ & 0.8213 & 0.5556 & 0.8940 & 0.6691 & & \\
\hline \multirow{3}{*}{8} & & $3.5 \mathrm{~V}$ & 0.6124 & 0.5556 & 0.8679 & 0.6039 & & \\
\hline & Frequency variance & $4.5 \mathrm{~V}$ & 0.6907 & 0.5556 & 0.7590 & 0.6164 & 0.6194 & 6 \\
\hline & & $6.0 \mathrm{~V}$ & 0.7157 & 0.5556 & 0.8978 & 0.6378 & & \\
\hline & & $3.5 \mathrm{~V}$ & 0.5675 & 0.5556 & 0.7430 & 0.5779 & & \\
\hline 9 & HHT marginal energy (HME) & $4.5 \mathrm{~V}$ & 0.4637 & 0.5556 & 0.8415 & 0.5566 & 0.5057 & 9 \\
\hline & & $6.0 \mathrm{~V}$ & 0.3360 & 0.3333 & 0.8192 & 0.3827 & & \\
\hline & & $3.5 \mathrm{~V}$ & 0.7513 & 0.7778 & 0.9545 & 0.7875 & & \\
\hline 10 & HHT marginal energy spectrum entropy (HMESE) & $4.5 \mathrm{~V}$ & 0.8404 & 1 & 0.9643 & 0.9486 & 0.8569 & 2 \\
\hline & & $6.0 \mathrm{~V}$ & 0.9094 & 0.7778 & 0.9518 & 0.8347 & & \\
\hline
\end{tabular}

the copula parameters. Figure 9 shows the system reliability curves of the motor using different copulas. To determine the best copula function, the AIC values of the five candidate copula families were obtained and compared. From Table 4, one can see that the AIC value of Frank copula function is the minimum, so it was adopted as the best choice among the candidates.

Figure 10 shows the marginal reliability and system reliability functions under both s-dependent and s-independent assumptions. One can see that the reliability function by assuming s-independent degradation features tends to be much lower than that based on the s-dependent assumption. Clearly, the dependence among the multiple features cannot be ignored.

\section{Conclusions}

This study is focused on the reliability modeling of vibrationbased ADT data. The proposed framework for data analysis
TABLE 4: Goodness-of-fit for the candidate copulas.

\begin{tabular}{lcc}
\hline Copula family & AIC value & Ranking \\
\hline Gaussian copula & 42961 & 5 \\
$t$-copula & -2278 & 2 \\
Clayton copula & -1179 & 3 \\
Frank copula & -17136 & 1 \\
Joe copula & -899 & 4 \\
\hline
\end{tabular}

focuses on feature selection and multivariate accelerated degradation modeling. A set of feature selection criteria were used to select the most suitable features after extracting features from raw vibration signals. Then, a multivariate s-dependent ADT model was presented, where Brownian motion was applied to establish accelerated degradation model for each feature and a copula function was employed to characterize the dependence among multiple degradation 


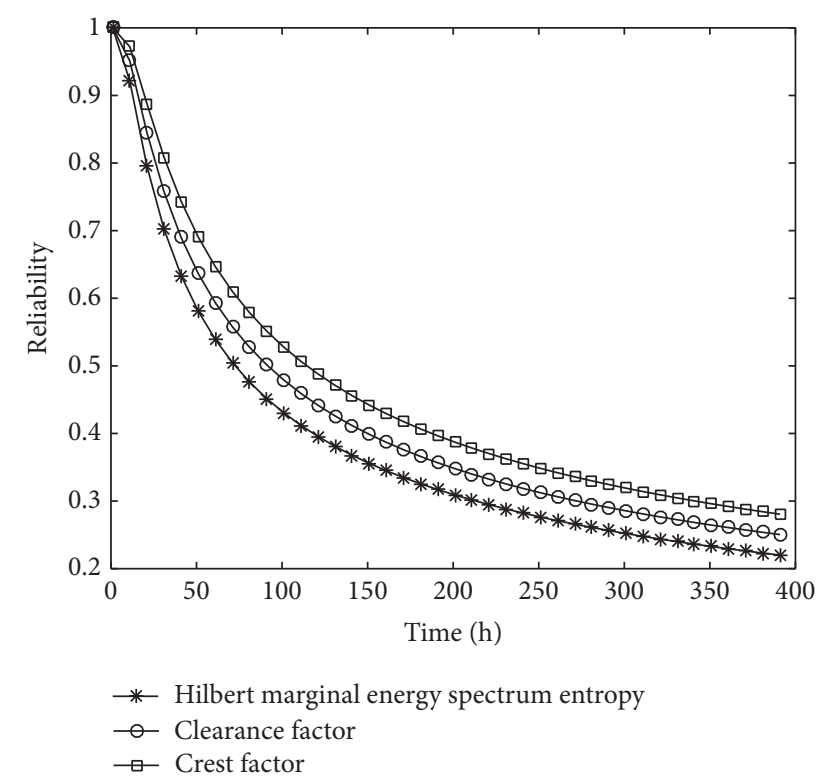

FIGURE 8: Reliability of three features under normal operating condition.

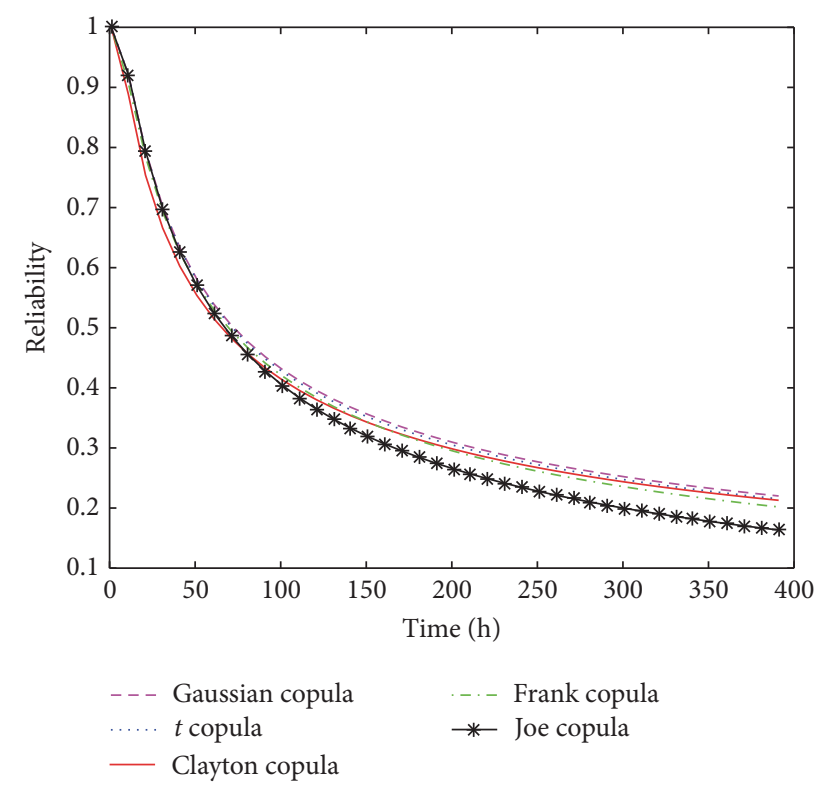

FIGURE 9: Comparison of different copula functions.

processes. A case study on motor vibration-based CSADT data was conducted to demonstrate the usefulness and validity of the proposed model and statistical method. The results show that the proposed framework can provide a powerful tool for modeling the reliability of products exhibiting multiple dependent degradation features.

\section{Competing Interests}

The authors declare that there is no conflict of interests regarding the publication of this paper.

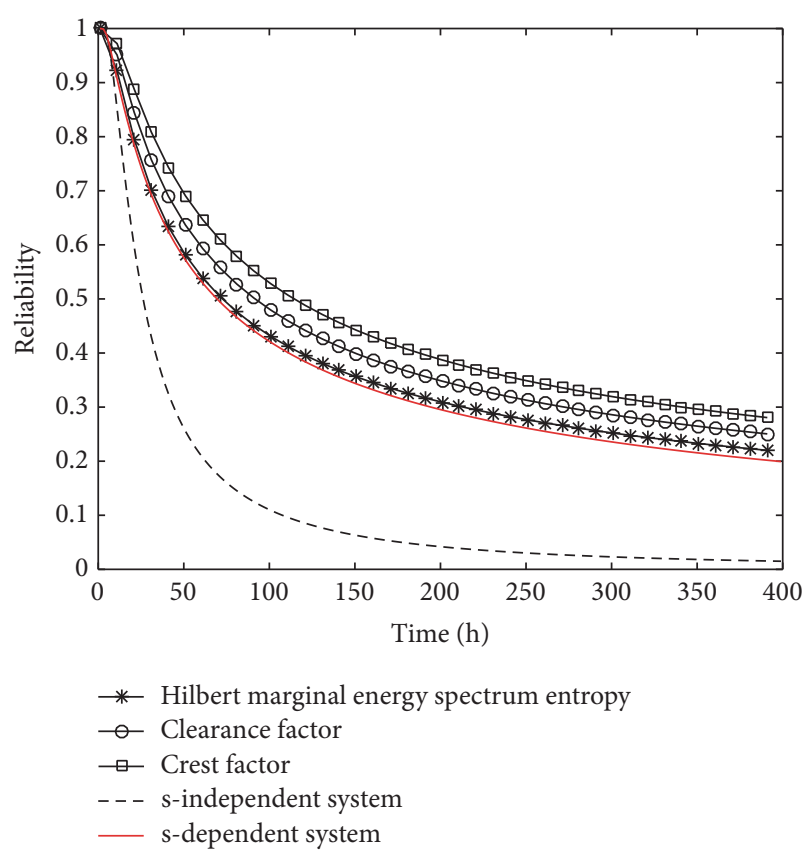

FIGURE 10: Comparison of the system reliability.

\section{Acknowledgments}

This work is partially supported by the National Natural Science Foundation of China (Grant nos. 61603018 and 61104182) and the China Scholarship Council. The work of Dr. Haitao Liao was partially supported by the U.S. National Science Foundation under Grant \#CMMI-1238304.

\section{References}

[1] S. Kumar and M. Pecht, "Modeling approaches for prognostics and health management of electronics," International Journal of Performability Engineering, vol. 6, no. 5, pp. 467-476, 2010.

[2] J. Lee, F. Wu, W. Zhao, M. Ghaffari, L. Liao, and D. Siegel, "Prognostics and health management design for rotary machinery systems-reviews, methodology and applications," Mechanical Systems and Signal Processing, vol. 42, no. 1-2, pp. 314-334, 2014.

[3] P. Nectoux, R. Gouriveau, K. Medjaher et al., "PRONOSTIA: an experimental platform for bearings accelerated degradation tests," in Proceedings of the IEEE International Conference on Prognostics and Health Management (PHM '12), pp. 1-8, Denver, Colo, USA, 2012.

[4] L. Liu, X.-Y. Li, W. Zhang, and T.-M. Jiang, "Fuzzy reliability prediction of rotating machinery product with accelerated testing data," Journal of Vibroengineering, vol. 17, no. 8, pp. 41934210, 2015.

[5] J. Lee, J. Ni, D. Djurdjanovic, H. Qiu, and H. Liao, "Intelligent prognostics tools and e-maintenance," Computers in Industry, vol. 57, no. 6, pp. 476-489, 2006.

[6] J. Coble and J. W. Hines, "Applying the general path model to estimation of remaining useful life," International Journal of Prognostics and Health Management, vol. 2, no. 1, pp. 71-82, 2011.

[7] B. Zhang, L. Zhang, and J. Xu, "Degradation feature selection for remaining useful life prediction of rolling element bearings," Quality and Reliability Engineering International, vol. 32, no. 2, pp. 547-554, 2016. 
[8] L. Liao, "Discovering prognostic features using genetic programming in remaining useful life prediction," IEEE Transactions on Industrial Electronics, vol. 61, no. 5, pp. 2464-2472, 2014.

[9] K. Javed, R. Gouriveau, N. Zerhouni, and P. Nectoux, "Enabling health monitoring approach based on vibration data for accurate prognostics," IEEE Transactions on Industrial Electronics, vol. 62, no. 1, pp. 647-656, 2015.

[10] F. Camci, K. Medjaher, N. Zerhouni, and P. Nectoux, "Feature evaluation for effective bearing prognostics," Quality and Reliability Engineering International, vol. 29, no. 4, pp. 477-486, 2013.

[11] K. Yang and G. Yang, "Performance degradation analysis using principal component method," in Proceedings of the Annual Reliability and Maintainability Symposium, pp. 136-141, Philadelphia, Pa, USA, January 1997.

[12] S. Lu, H. Lu, and W. J. Kolarik, "Multivariate performance reliability prediction in real-time," Reliability Engineering \& System Safety, vol. 72, no. 1, pp. 39-45, 2001.

[13] R. B. Nelsen, An Introduction to Copulas, Springer Science \& Business Media, New York, NY, USA, 2nd edition, 2007.

[14] J. K. Sari, M. J. Newby, A. C. Brombacher, and L. C. Tang, "Bivariate constant stress degradation model: LED lighting system reliability estimation with two-stage modelling," Quality and Reliability Engineering International, vol. 25, no. 8, pp. 10671084, 2009.

[15] Z. Q. Pan, N. Balakrishnan, and Q. Sun, "Bivariate constantstress accelerated degradation model and inference," Communications in Statistics-Simulation and Computation, vol. 40, no. 2, pp. 247-257, 2011.

[16] W. Peng, Y.-F. Li, Y.-J. Yang, S.-P. Zhu, and H.-Z. Huang, "Bivariate analysis of incomplete degradation observations based on inverse Gaussian processes and copulas," IEEE Transactions on Reliability, vol. 65, no. 2, pp. 624-639, 2016.

[17] Z. Liu, X. Ma, J. Yang, and Y. Zhao, "Reliability modeling for systems with multiple degradation processes using inverse Gaussian process and copulas," Mathematical Problems in Engineering, vol. 2014, Article ID 829597, 10 pages, 2014.

[18] Y. Wang and H. Pham, "Modeling the dependent competing risks with multiple degradation processes and random shock using time-varying copulas," IEEE Transactions on Reliability, vol. 61, no. 1, pp. 13-22, 2012.

[19] X. Li and P. Xue, "Multivariate storage degradation modeling based on copula function," Advances in Mechanical Engineering, vol. 2014, Article ID 503407, 7 pages, 2014.

[20] Z.-S. Ye and M. Xie, "Stochastic modelling and analysis of degradation for highly reliable products," Applied Stochastic Models in Business and Industry, vol. 31, no. 1, pp. 16-32, 2015.

[21] H. Liao and E. A. Elsayed, "Reliability inference for field conditions from accelerated degradation testing," Naval Research Logistics, vol. 53, no. 6, pp. 576-587, 2006.

[22] Q. Guan, Y. C. Tang, and A. C. Xu, "Objective Bayesian analysis accelerated degradation test based on Wiener process models," Applied Mathematical Modelling, vol. 40, no. 4, pp. 2743-2755, 2016.

[23] J. Lin, M. J. Zuo, and K. R. Fyfe, "Mechanical fault detection based on the wavelet de-noising technique," Journal of Vibration and Acoustics, vol. 126, no. 1, pp. 9-16, 2004.

[24] D. L. Donoho, "De-noising by soft-thresholding," IEEE Transactions on Information Theory, vol. 41, no. 3, pp. 613-627, 1995.

[25] D. Goyal and B. S. Pabla, "Condition based maintenance of machine tools-a review," CIRP Journal of Manufacturing Science and Technology, vol. 10, pp. 24-35, 2015.
[26] N. E. Huang and Z. Wu, "A review on Hilbert-Huang transform: method and its applications to geophysical studies," Reviews of Geophysics, vol. 46, no. 2, Article ID RG2006, 2008.

[27] L. A. Escobar and W. Q. Meeker, "A review of accelerated test models," Statistical Science, vol. 21, no. 4, pp. 552-577, 2006.

[28] G. K. Bhattacharyya and A. Fries, "Fatigue failure modelsBirnbaum-Saunders vs. Inverse Gaussian," IEEE Transactions on Reliability, vol. 31, no. 5, pp. 439-441, 1982.

[29] A. Sklar, "Fonctions de répartition à $\mathrm{n}$ dimensions et leurs marges," Publications de l'Institut de Statistique de l'Université de Paris, no. 8, pp. 229-231, 1959.

[30] H. Joe, Multivariate Models and Multivariate Dependence Concepts, CRC Press, Boca Raton, Fla, USA, 1st edition, 1997.

[31] H. Joe, "Asymptotic efficiency of the two-stage estimation method for copula-based models," Journal of Multivariate Analysis, vol. 94, no. 2, pp. 401-419, 2005. 


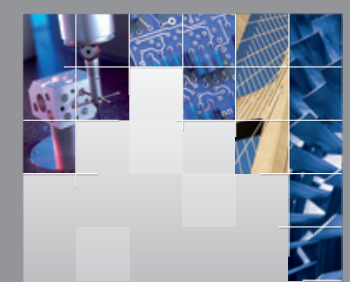

\section{Enfincering}
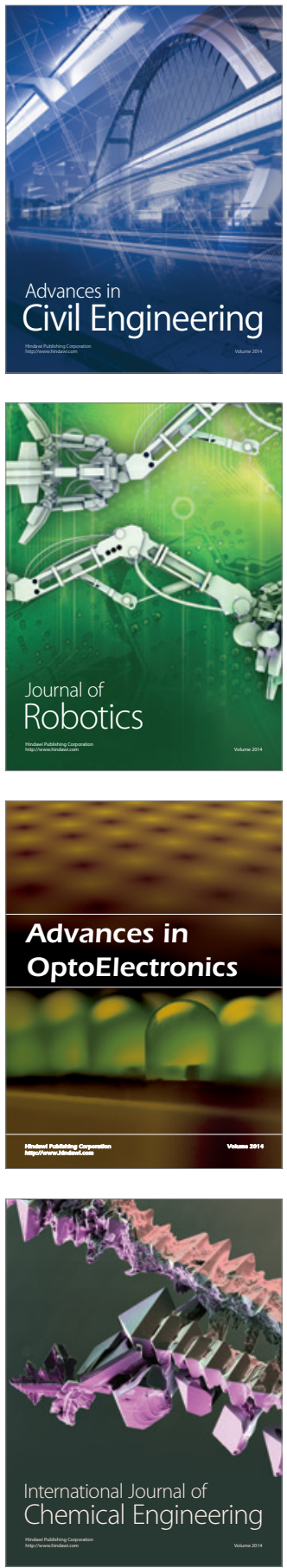

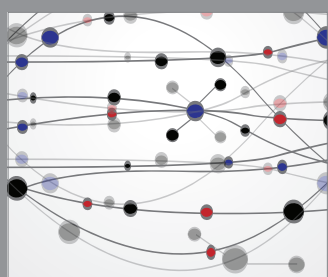

The Scientific World Journal

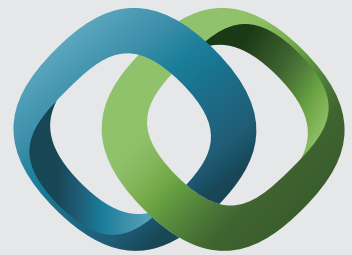

\section{Hindawi}

Submit your manuscripts at

http://www.hindawi.com
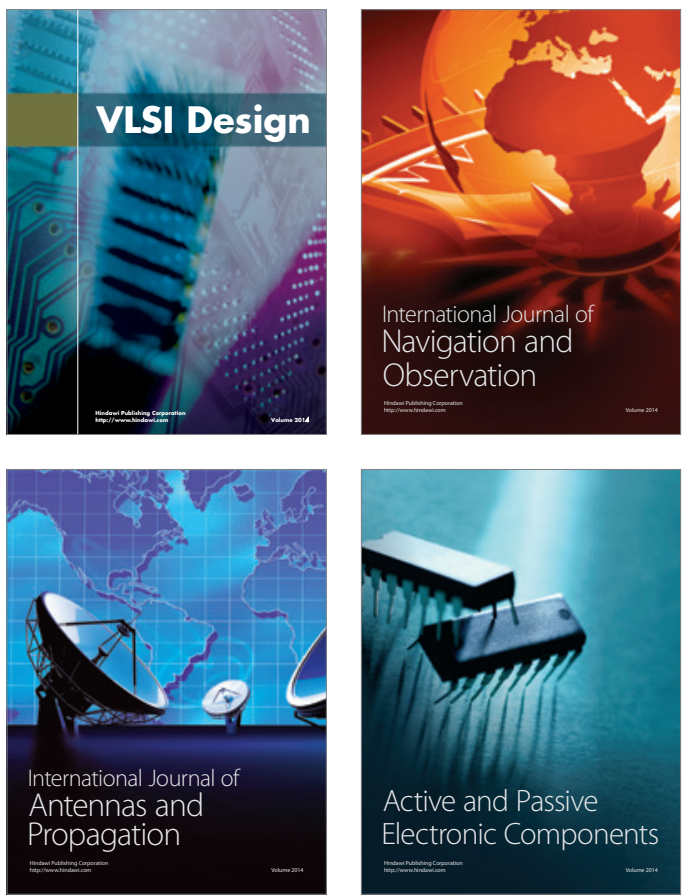
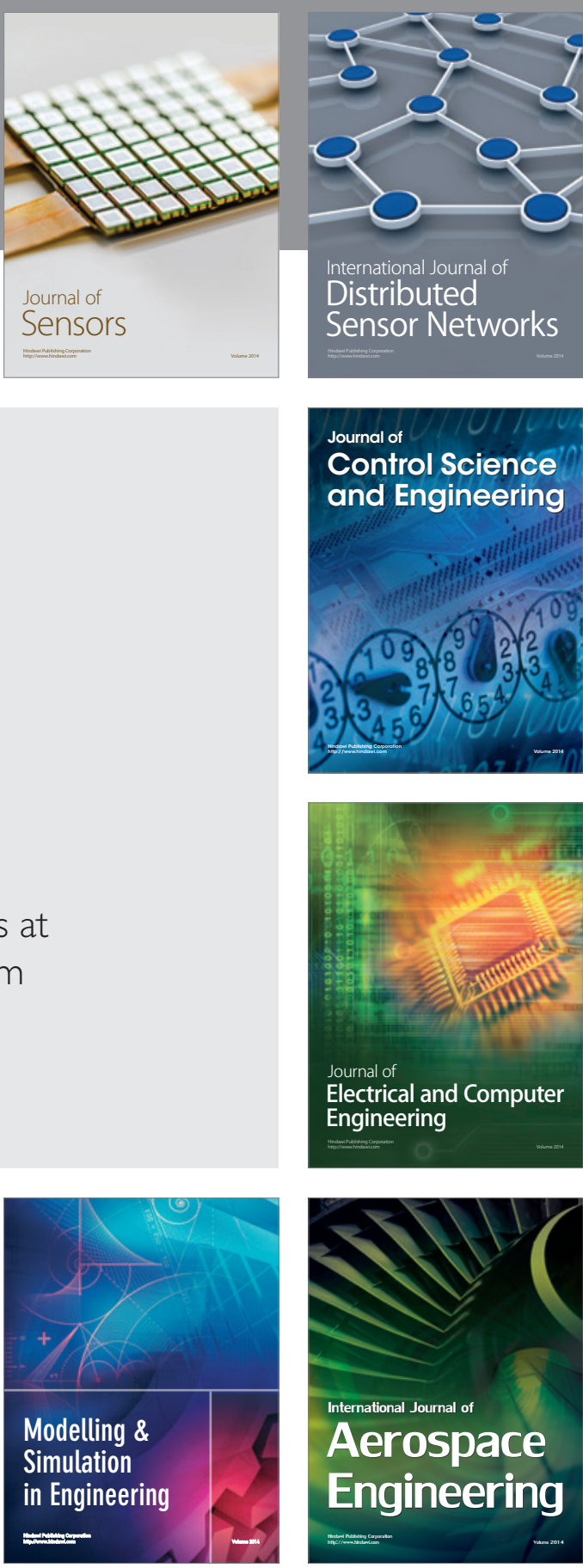

International Journal of

Distributed

Sensor Networks

Journal of

Control Science

and Engineering
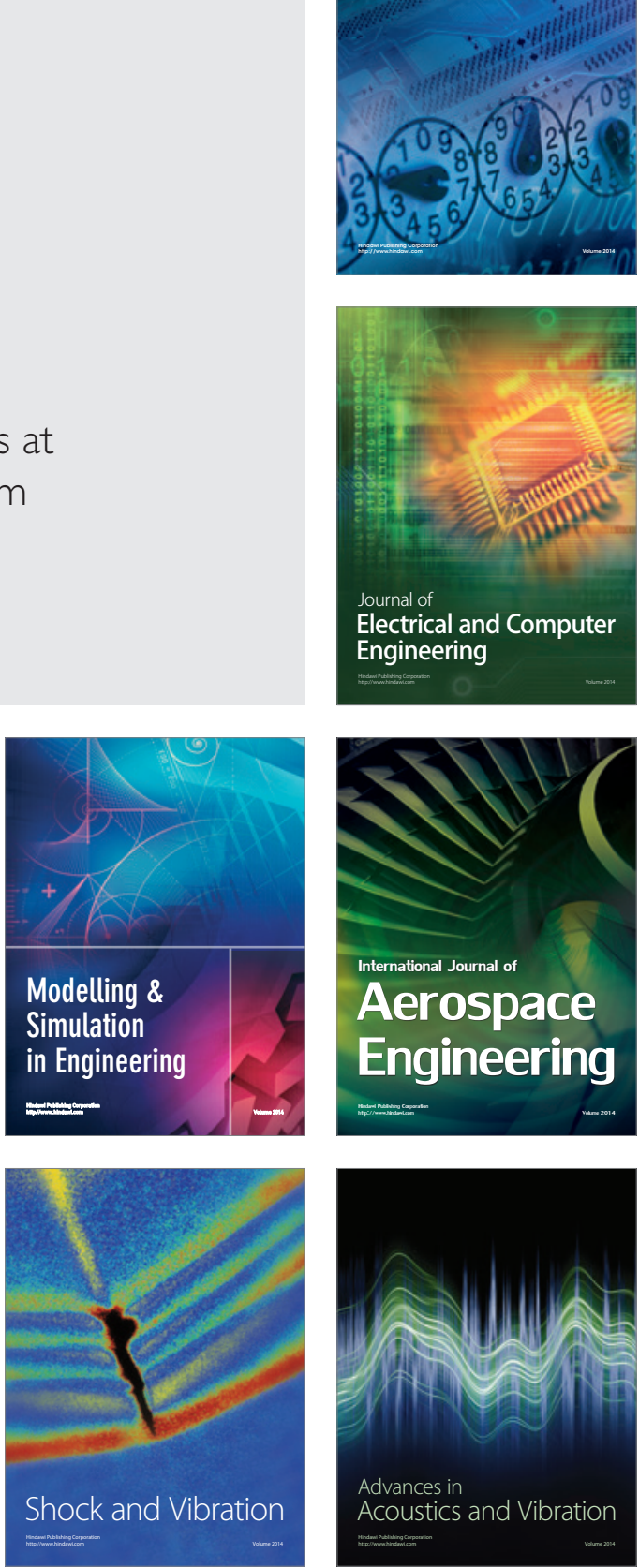